\title{
Risk Factors for Hemoptysis in Pulmonary Tuberculosis Patients from Southern China: A Retrospective Study
}

\author{
Shouyong Tan ${ }^{*}$, Danxiong Sun ${ }^{1,2}$, Tiantuo Zhang3, Yanqiong $\mathrm{Li}^{1}$, Yuanyuan $\mathrm{CaO}^{4}$, \\ Moses M. Njire ${ }^{4}$, Changwei Wang ${ }^{4}$, Tianyu Zhang ${ }^{*}$ \\ ${ }^{1}$ State Key Laboratory of Respiratory Diseases, Department of Internal Medicine, The Guangzhou Chest \\ Hospital, Guangzhou, China \\ ${ }^{2}$ Respiratory Department, The First People Hospital of Yunnan Province, Kunming, China \\ ${ }^{3}$ Department of Internal Medicine, The Third Affiliated Hospital of Sun Yat-sen University, Guangzhou, \\ China \\ ${ }^{4}$ State Key Laboratory of Respiratory Diseases, Guangzhou Institutes of Biomedicine and Health, Chinese \\ Academy of Sciences, Guangzhou, China \\ Email: tanshyo@163.com, ${ }^{*}$ zhang tianyu@gibh.ac.cn
}

Received 8 October 2014; revised 9 November 2014; accepted 18 November 2014

Copyright (C) 2014 by authors and Scientific Research Publishing Inc.

This work is licensed under the Creative Commons Attribution International License (CC BY). http://creativecommons.org/licenses/by/4.0/

(c) (i) Open Access

\section{Abstract}

Clinical characteristics of tuberculosis (TB) patients from southern China with pulmonary tuberculosis hemoptysis (PTH) were analyzed retrospectively in order to improve the diagnosis of TB, reduce mortality and prevent the transmission of TB. A total of 1227 cases of pulmonary TB patients hospitalized in the Third Affiliated Hospital of Sun Yat-sen University and Guangzhou Chest Hospital from January to December of 2011 were analyzed retrospectively. 1) The male/female ratio of the 1227 tuberculosis cases was $2.15: 1$. There were 403 cases $(32.8 \%)$ of PTH with a male/ female ratio of 3.03:1. 2) The ratio of patients with PTH to those with TB was designated as Rh. The $\mathrm{Rh}$ in the male group $(36.2 \%, 303$ cases) was higher than that in the female group $(25.6 \%, 100$ cases, risk ratio $(R R)=1.41, P \leq 0.001) .3$ ) The $R h$ in the elderly group ( $\geq 60$ years old, $20.3 \%, 56$ cases) was lower than that in the younger patients group $(20-39$ years old, 45.4\%, 189 cases, $R R=$ $2.51, P \leq 0.001) .4)$ The $R h$ in initial treatment group $(29.6 \%, 296$ cases) was lower than that in the retreatment group $(46.9 \%, 107$ cases, $R R=1.58, P \leq 0.001) .5)$ The $R h$ in sputum-positive TB patients $(44.5 \%, 297$ cases) was significantly higher than that in the smear-negative TB patients $(18.9 \%, 106$ cases, $R R=2.35, P \leq 0.001) .6)$ The $R h$ of patients with lung lesions range $<3$ lung fields $(31.7 \%, 105$ cases) was not significantly different with that of patients with lung lesions range $\geq 3$ lung fields $(33.3 \%, 298$ cases, $R R=1.05, P=0.96>0.05)$. 7$)$ The $R$ of patients with cavities $(51.8 \%, 309$ cases) was higher than that of patients without cavities $(14.9 \%, 94$ cases, $R R=$ $3.48, P \leq 0.001$ ). Male, young, retreated, sputum-positive TB patients and those with cavitary TB

\footnotetext{
${ }^{*}$ Corresponding authors.
}

How to cite this paper: Tan, S.Y., Sun, D.X., Zhang, T.T., Li, Y.Q., Cao, Y.Y., Njire, M.M., Wang, C.W. and Zhang, T.Y. (2014) Risk Factors for Hemoptysis in Pulmonary Tuberculosis Patients from Southern China: A Retrospective Study. Journal of Tuberculosis Research, 2, 173-180. http://dx.doi.org/10.4236/jtr.2014.24022 
were more predisposed to PTH in southern China. TB patients with such characteristics should be sensitized and accorded good care.

\author{
Keywords
}

Tuberculosis, Hemoptysis, Risk Factors

\title{
1. Introduction
}

Hemoptysis is the expectoration or spitting of blood originating from any part of the respiratory tract, usually from hemorrhage in the lung parenchyma (pulmonary alveoli) and the bronchial arteries [1]. It may be a symptom of several diseases, more or less severe, but its appearance induces concern to the patient and requires a full diagnostic investigation. Bronchiectasis, pulmonary tuberculosis (TB) and lung cancer are the main causes of hemoptysis. The frequency of each disease as a cause of hemoptysis varies in different series according to the geographical area. Pulmonary TB is an important cause of hemoptysis in developing countries [2], whereas, in developed countries, bronchiectasis, lung cancer and bronchitis are the main causes. TB may cause hemoptysis either in active state (cavitary lesions, rupture of pulmonary artery aneurysms) or as a late sequelae (rupture of aneurysms or secondary to bronchiectasis). Rupture of Rasmussen's aneurysm can occur with active disease or as a late finding. It occurs when there is rupture of ectatic portions of the pulmonary arteries traversing thickwalled cavities [3].

Kralingen et al. [4] from Nepal have reported 63 cases of hemoptysis patients, among which the most common (65\%) patients were infected with TB and its sequelae, followed by pneumonia (17\%), bronchitis (13\%) and lung cancer (5\%). However, TB is not the main cause of hemoptysis any more in some medium-level developed countries. Fidan et al. [4] from Turkey reported 108 cases of hemoptysis with lung cancer as the most common cause $(34.3 \%)$, followed by bronchiectasis $(25.0 \%)$, TB (17.6\%), pneumonia $(10.2 \%)$ and pulmonary embolism (4.6\%). In developed countries, bronchiectasis, lung cancer and bronchitis have been the main cause of hemoptysis mainly due to the good control of TB. In other studies, Porzezińska et al. [5] from Poland retrospectively analyzed 431 cases of hemoptysis patients with tumor as the most common cause (40.4\%). Tsoumakidou et al. [6] analyzed 184 cases of hemoptysis from Greece and found bronchiectasis (26\%) and chronic bronchitis $(23 \%)$ as the main causes followed by acute bronchitis $(15 \%)$ and lung cancer $(13 \%)$. TB is still the main cause of hemoptysis in some developed countries. For example, Pires et al. [7] reported 221 adult hemoptysis patients cases from Portugal and found that the most common cause was TB sequelae (22.2\%), followed by bronchiectasis (15.8\%), lung cancer (13.9\%), TB (10\%) and pneumonia (4.5\%). China is a developing country with high incidence of TB with pulmonary TB being the main cause of hemoptysis.

Typical symptoms of TB may include cough, sputum, hemoptysis companied with anorexia, weakness, night sweat, chills and fever. The CT image for TB is usually not obvious even with enhanced scan, and most patients show slight hemoptysis while others could have moderate and even severe hemoptysis [8]. A recent study in China reported hemoptysis as the second cause of death in pulmonary TB patients [9]. Hemoptysis mainly results in asphyxiation and sometimes causes hemorrhagic shock or both. Pulmonary tuberculosis hemoptysis (PTH) can also cause complications, such as TB dissemination and pulmonary atelectasis. In addition, patients with PTH may transmit TB more easily, as PTH patients have a higher $M$. tuberculosis-positive rate [10], which has also been reported in this study. The M. tuberculosis-positive pulmonary TB patients are infectious TB patients. Therefore, we analyzed the clinical characteristics of PTH patients in southern China in order to improve the diagnosis and treatment of PTH and to prevent the transmission of TB.

\section{Materials and Methods}

\subsection{Ethical Approval}

Individual participants gave written informed consent before enrollment in the study. All the patient information was routinely collected and recorded by attending physicians. All patient procedures were reviewed and approved by the Ethics Committees of Guangzhou Chest Hospital and the Third Affiliated Hospital of Sun Yat-sen University. 


\subsection{Setting and Study Population}

A total of 1227 cases of TB patients hospitalized at the Guangzhou Chest Hospital and the Third Affiliated Hospital of Sun Yat-sen University from January to December of 2011 were analyzed retrospectively. Guangzhou Chest Hospital is the largest TB treatment center in southern China, which admits about 70,000 patients/year while the latter is a general hospital, with about 5000 patients' admissions with respiratory diseases/year. All patients were initially treated as inpatients, but some persons were followed on an outpatient basis after hospitalization. Medical records from in-patient and out-patient treatments were reviewed for patients' demographics, TB treatment history, comorbidities, chest radiographs, smear test and M. tuberculosis cultivation. Patients were enrolled as they needed to be hospitalized and with clear demographic records. Patients with indeterminate test results, missing data, and data outliers were excluded. All the participants in this study were Han race.

\subsection{Criteria and Definitions}

1. Inclusion criteria for TB: Compliance with active TB (hereinafter referred to as TB) patients. Exclusion criteria for TB: TB patients with one of the following diseases: lung cancer, nontuberculosis mycobacteria bronchiectasis, pulmonary aspergillosis and non-tuberculosis mycobacteria lung disease.

2. TB diagnostic criteria: This was based on the TB diagnosis and treatment guidelines [11].

(1) M. tuberculosis-positive pulmonary TB indicates those that meet one of the following 4 criteria: sputum smear microscopy for acid-fast bacilli positive twice; sputum smear microscopy for acid-fast bacilli positive once and active TB lesion showed in chest radiographs; sputum smear microscopy for acid-fast bacilli positive once and $M$. tuberculosis culture positive once; sputum smear microscopy for acid-fast bacilli negative but $M$. tuberculosis culture positive once and active TB lesion showed in chest radiographs.

(2) Smear-positive pulmonary TB indicates those that meet one of the following 3 criteria: sputum smear microscopy for acid-fast bacilli positive twice; sputum smear microscopy for acid-fast bacilli positive once and active TB lesion showed in chest radiographs. In short, M. tuberculosis-positive pulmonary TB indicates smearpositive or M. tuberculosis culture-positive; smear-positive pulmonary TB means smear-positive only.

(3) M. tuberculosis-negative pulmonary TB is sputum smear microscopy for acid-fast bacilli negative three times, M. tuberculosis culture negative once and meet 3 items of the following criteria 1) to 6) or either of 7) or 8). 1) Typical clinical symptoms of TB and chest X-ray findings; 2) Anti-tuberculosis treatment is effective; 3) Other non-tuberculosis lung diseases were excluded clinically; 4) PPD (5IU) test is strongly positive and serum anti-tuberculosis antibodies are positive; 5) Sputum M. tuberculosis PCR test and probe detection are positive; 6) TB confirmed by extra-pulmonary pathology; 7) Acid-fast mycobacteria is detected in bronchial alveolus lavage (BAL) fluid; 8) Bronchus or lung histopathology was confirmed with TB lesions.

(4) Initial treated pulmonary TB was confirmed with one of the following criteria: 1) Never used anti-tuberculosis drug before; 2) Failed to complete the first course of anti-tuberculosis treatment; 3) Irregular chemotherapy less than a month.

(5) Retreatment TB was confirmed with one of the following criteria: 1) Initial treatment failed; 2) Recurrence after completion of regular chemotherapy or directly observed treatment short-course (DOTS); 3) New case but with irregular chemotherapy more than a month; 4) New lesions emerged or recurrence or deterioration of the rudimental lesion after pulmonary resection.

3. Please refer to the clinical TB diagnosis and treatment guidelines [12] for defining the index of hemoptysis. 1) Small hemoptysis: $<100 \mathrm{ml} / 24 \mathrm{hr}$; 2) Middle hemoptysis: $\geq 100 \mathrm{ml}$ and $<500 \mathrm{ml} / 24 \mathrm{hr}$, or $100-300 \mathrm{ml} / \mathrm{hr}$; 3) Heavy hemoptysis: $>500 \mathrm{ml} / 24 \mathrm{hr}$ or $>300 \mathrm{ml} / \mathrm{hr}$. Here the volume of hemoptysis was calculated according to biggest volume during a patient's course of disease.

4. Exclusion criteria for PTH: Smear and culture were negative and the symptom got better after anti-inflamation treatment for 2 weeks.

\subsection{Statistical Analysis}

Statistical analysis was performed using SPSS 14.0 statistical software (SPSS, Chicago). For qualitative data, $\chi^{2}$ test was used. $\mathrm{U}$ test was used to compare the sample rate and the overall rate. $\mathrm{P}<0.05$ was considered statistically significant. 


\section{Results}

\subsection{Demographic Characteristics of PTH Patients}

Among the 1227 cases of pulmonary TB patients recruited in this study, 837 were males and 390 were females with a ratio of 2.14. Their gender and age distribution is showed in Table 1. A total of 403 cases were PTH positive. Male patients had higher Rh (the ratio of patients with PTH/those with TB within the same group, 36.2\%) than that of female patients $(25.6 \%)$ with a risk ratio $(\mathrm{RR})=1.41, \mathrm{P} \leq 0.001$. Most PTH patients fell into the 20 $\leq$ age $\leq 39$ group $(46.9 \%$, Table 1$)$ and the number of PTH patients decreased continuously along with the increasing age ( $>20$, Figure 1 (a)). TB patients also had a relatively higher $\mathrm{Rh}$ at the $20 \leq$ age $\leq 39$ group (45.4\%, 189 cases) than the older patients at the $\geq 60$ age group $(20.3 \%, 56$ cases, $\mathrm{RR}=2.51, \mathrm{P} \leq 0.001)$. The $\mathrm{Rh}$ declined continuously along with patients' increased ages starting from age 20 (Figure 1(b)).

There were 244 cases $(60.55 \%)$ of small hemoptysis, 126 cases $(31.27 \%)$ of middle hemoptysis and 33 cases $(8.19 \%)$ of heavy hemoptysis. The constituent ratio of patients with small hemoptysis was significantly higher than that of patients with middle hemoptysis $\left(\chi^{2}=29.313, \mathrm{P}<0.001\right)$ and that of patients with heavy hemoptysis $\left(\chi^{2}=14.292, \mathrm{P}<0.001\right)$. The constituent ratios of patients with middle hemoptysis and patients with heavy hemoptysis showed no statistical significance. Among the PTH patients, there were 94 cases $(23.33 \%)$ combined with TB bronchiectasis and 75 cases (18.61\%) combined with endobronchial TB.

\section{Table 1. Demographic data of 1227 TB patients.}

\begin{tabular}{|c|c|c|c|c|c|c|}
\hline & & \multicolumn{2}{|c|}{ Pulmonary TB } & \multirow{2}{*}{$\begin{array}{l}\text { Risk ratio } \\
\text { (RR) }\end{array}$} & \multirow{2}{*}{$95 \% \mathrm{CI}$} & \multirow{2}{*}{$P$ value } \\
\hline & & Hemoptysis (\%) $\mathrm{N}=403$ & No hemoptysis (\%) $\mathrm{N}=824$ & & & \\
\hline \multirow{2}{*}{ Sex } & Male & $303(75.2)$ & $534(64.8)$ & 1.41 & \multirow{2}{*}{$1.17-1.70$} & \multirow{2}{*}{$<0.001$} \\
\hline & Female & $100(24.8)$ & $290(35.2)$ & Reference & & \\
\hline \multirow{4}{*}{$\begin{array}{c}\text { Age } \\
\text { (years) }\end{array}$} & $\leq 19$ & $44(10.9)$ & $81(9.8)$ & & \multirow[b]{2}{*}{$1.98-3.17$} & \multirow[b]{2}{*}{$<0.001$} \\
\hline & $20-39$ & $189(46.9)$ & $227(27.6)$ & 2.51 & & \\
\hline & $40-59$ & $114(28.3)$ & $263(31.9)$ & 1.67 & $1.28-2.19$ & $<0.001$ \\
\hline & $\geq 60$ & $56(13.9)$ & $253(30.7)$ & Reference & & \\
\hline
\end{tabular}

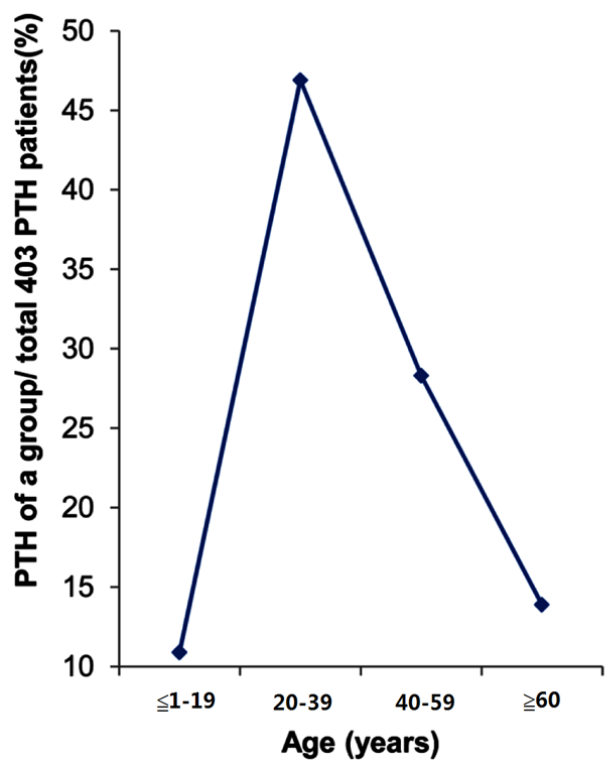

(a)

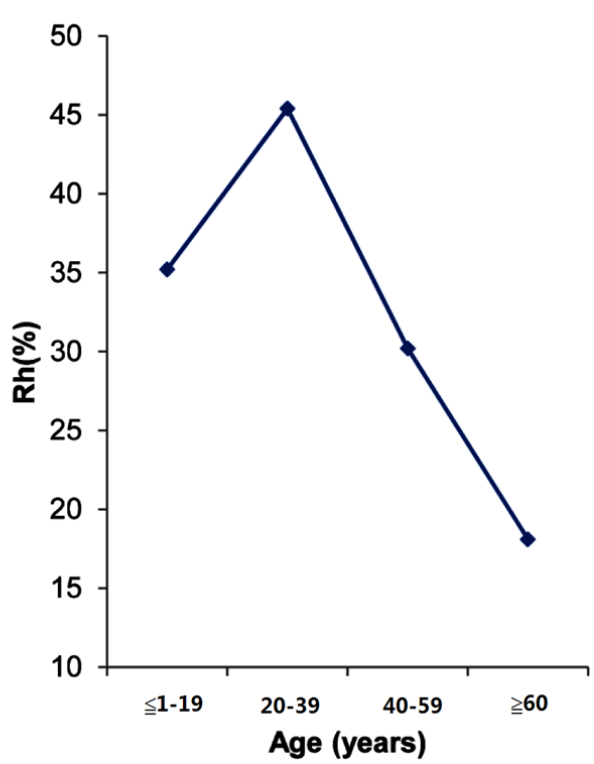

(b)

Figure 1. a) PTH patients' age distribution. The ratio = number of PTH patients in the subgroup/403 (the total number of PTH patients). b) Rh in different PTH patients' age groups. 


\subsection{Treatment History and Sputum Bacteriological Characteristics of PTH Patients}

The treatment history and sputum bacteriological characteristics of the 1227 pulmonary TB patients are shown in Table 2. Among the 999 (81.4\%) pulmonary TB patients in the initial treatment group, there were 296 cases of PTH $(\mathrm{Rh}=29.6 \%)$. There were 107 cases of PTH $(\mathrm{Rh}=46.9 \%)$ among the $228(18.6 \%)$ pulmonary TB patients in the retreatment group. The $\mathrm{Rh}$ in the latter group was significantly higher than that in the former one $\left(\chi^{2}\right.$ $=25.189, \mathrm{P}<0.001)$.

There were 667 sputum-positive TB patients $(54.4 \%)$ including $297 \mathrm{PTH}(\mathrm{Rh}=44.5 \%)$ and 560 smear-negative TB patients $(45.6 \%)$ including $106 \mathrm{PTH}(\mathrm{Rh}=18.9 \%)$. The $\mathrm{Rh}$ of sputum-positive PTH patients was significantly higher than that of the sputum-negative PTH patients $\left(\chi^{2}=40.691, \mathrm{P}<0.001\right)$.

\subsection{Chest Radiographic Characteristics of PTH Patients}

The chest radiographic results of all the 1227 pulmonary TB patients are summarized in Table 3 . There were $331(27.0 \%)$ TB patients with lung lesion range $<3$ lung fields, including $105 \mathrm{PTH}$ patients $(\mathrm{Rh}=31.7 \%)$. TB patients with lung lesion range $\geq 3$ lung fields were $896(73.0 \%)$, including $298 \mathrm{PTH}$ patients $(\mathrm{Rh}=33.3 \%)$. The $\mathrm{Rh}$ in these 2 groups showed no significant difference, i.e., there was no significant correlation between scope of pulmonary lesions and hemoptysis $\left(\chi^{2}=2.588, \mathrm{P}>0.10\right)$. However, Rh in pulmonary TB patients with cavities is much higher than that in those without cavities $\left(\chi^{2}=189.71, \mathrm{P}<0.001\right)$.

\section{Discussion}

There were relatively more hospitalized male TB patients than females (2.15:1). Various reasons could possibly explain this difference: firstly, men are more exposed to environments contaminated with M. tuberculosis because of their relatively higher mobility to the work stations and other social places. On the other hand, women are less mobile as they are often confined to family household chores, thus less exposed to M. tuberculosis. Secondly, women occupy the lower socio-economic cadres in the society, and thus not many could be easily found in the hospitals. Thirdly, the pathogenecity and virulence of M. tuberculosis in humans may be related to gender, in which men may be more vulnerable to TB than women due to less competent immune potency [13]. Fourthly, the fact that more smokers are males could partially account for more male TB patients than females. In fact, recent studies have described an association between tobacco smoke and the risk of developing TB [14] [15]. This study also showed that there were more male PTH patients (3.03:1) than there were females, similar to other studies reported in the literature [16].

Table 2. 1227 cases of early retreatment TB patients and bacteriological conditions.

\begin{tabular}{|c|c|c|c|c|c|c|}
\hline & & \multicolumn{2}{|c|}{ Pulmonary TB } & \multirow{2}{*}{$\begin{array}{l}\text { Risk ratio } \\
\text { (RR) }\end{array}$} & \multirow{2}{*}{$95 \%$ CI } & \multirow[b]{2}{*}{$\mathrm{P}$ value } \\
\hline & & $\begin{array}{l}\text { Hemoptysis }(\%) \\
\qquad N=403\end{array}$ & $\begin{array}{l}\text { No hemoptysis (\%) } \\
\qquad \mathrm{N}=824\end{array}$ & & & \\
\hline \multirow{2}{*}{$\begin{array}{c}\text { History of } \\
\text { pulmonary TB }\end{array}$} & Initial treatment & $296(73.4)$ & $703(70.4)$ & Reference & & \\
\hline & Retreatment & $107(26.6)$ & $121(29.6)$ & 1.58 & $1.32-1.90$ & $<0.001$ \\
\hline \multirow{2}{*}{$\begin{array}{c}\text { Sputum } \\
\text { bacteriology }\end{array}$} & Smear-positive TB & $297(73.7)$ & $370(44.9)$ & 2.35 & $1.97-2.81$ & $<0.001$ \\
\hline & Smear-negative TB & $106(26.3)$ & $454(55.1)$ & Reference & & \\
\hline
\end{tabular}

Table 3. Imageological change of 1227 cases with TB.

\begin{tabular}{|c|c|c|c|c|c|c|}
\hline & & \multicolumn{2}{|c|}{ Pulmonary TB } & \multirow[b]{2}{*}{ Risk ratio } & \multirow[b]{2}{*}{$95 \% \mathrm{CI}$} & \multirow[b]{2}{*}{$P$ value } \\
\hline & & $\begin{array}{l}\text { Hemoptysis }(\%) \\
\qquad \mathrm{N}=403\end{array}$ & $\begin{array}{l}\text { No hemoptysis (\%) } \\
\qquad N=824\end{array}$ & & & \\
\hline \multirow{2}{*}{ Lesion range } & $<3$ lung fields & $105(26.1)$ & $226(27.4)$ & Reference & \multirow[b]{2}{*}{$0.87-1.26$} & \multirow[b]{2}{*}{0.96} \\
\hline & $\geq 3$ lung fields & $298(73.9)$ & $598(72.6)$ & 1.05 & & \\
\hline \multirow{2}{*}{ Cavity } & Cavity & $309(79.6)$ & $287(34.8)$ & 3.48 & \multirow{2}{*}{$2.91-4.16$} & \multirow{2}{*}{$<0.001$} \\
\hline & No cavity & $94(20.4)$ & $537(65.2)$ & Reference & & \\
\hline
\end{tabular}


This study also showed that age was possibly an important independent risk factor for PTH, which was consistent with findings from the U.S. by Achkar et al. [17]. Patients with $20 \leq$ age $\leq 39$ were more prone to hemoptysis which could be attributed to the following reasons: firstly, such patients did not pay enough attention to respiratory symptoms, such as cough and sputum [8]. Most patients could not have been aware of the severity of hemoptysis until it happened, after which they sought medical attention. On the other hand, the elderly people undergo regular health examinations for age-related diseases, and thus the probable earlier detection and treatment of lung diseases reducing the incidence of hemoptysis. Secondly, immunity difference between the young and the elderly TB patients could also explain the findings [18]. People with hypoimmunity are more susceptible to TB; however, strong immune response is a double-edged sword and could also be detrimental. The human immune system attenuates with age with the lymphocytes becoming weaker in their function [19].

Heavy and middle hemoptysis are mainly observed in bronchiectasis, TB and relatively rare in other diseases. Studies from different geographical regions have reported different causes for hemoptysis. In China, Yuan et al. [20] reported heavy and middle hemoptysis in both bronchiectasis and TB patients, with middle hemoptysis also occurring in pulmonary TB patients and in low numbers in lung cancer and pneumonia patients. However, Reechaipichitkul et al. [3] from Thailand reported 101 cases of hemoptysis of which bronchiectasis was the main cause (33.7\%), followed by active TB (20.8\%) and then lung cancer (10.9\%). Pulmonary TB mainly results to heavy and middle hemoptysis because the TB lesions usually block the blood vessels carrying blood under high pressure resulting in breakage which can also result in ruptured Rasmussen's aneurysm [21] [22]. Wu et al. [23] reported 62 cases of heavy hemoptysis which were all associated with systemic circulation-pulmonary circulation shunt. In cases of chronic lung infections, tumors, pulmonary embolism and congenital heart and lung diseases, the blood in the pulmonary artery decreases. Therefore, compensatory hyperplasia of bronchial arteries and anastomosis bronchiectasis increases blood in pulmonary circulation resulting into a systemic circulationpulmonary circulation shunt. This is a common phenomenon in pulmonary TB patients which make them have heavy and middle hemoptysis. Here we showed that male gender and pulmonary TB were the main risk factors for heavy and middle hemoptysis, which is consistent with an earlier report from Turkey which indicated TB as an independent predictor of heavy hemoptysis [24].

In this study, hemoptysis accounted for $46.9 \%$ of retreated TB patients, and the corresponding Rh was significantly higher than that of the initial treatment TB group. Firstly, the reason could be that pulmonary TB in the retreated group had complications, such as bronchiectasis and pulmonary aspergillosis. Bronchiectasis results from chronic fibro-cavitary TB while pulmonary aspergillosis results from TB cavities being prone to secondary Aspergillus infection. Secondly, TB patients had attenuated immune function. Therefore, the decontamination function of bronchial epithelium was weakened, which was helpful for the fungi at the pars oralis pharyngis invading downward into lung tissues easily. Thirdly, rifamycins, aminoglycosides, quinolones and other broadspectrum anti-TB drugs could kill both $M$. tuberculosis and other bacteria and easily lead to dysbacteriosis and then eventual secondary pulmonary fungal infection [25].

TB cavities usually results from liquefied caseous necrotic lesions. Most TB cavities have collagen and cheese as the outer and inner layers respectively, and granulation tissues containing a lot of capillaries in between them. There is incomplete thrombosis in the blood vessels in the middle layer, which can be disrupted by TB infection leading to hemoptysis and eventually heavy hemoptysis. Most TB cavities have Rasmussen's aneurysms and their rupture often lead to middle and even heavy hemoptysis. For TB patients without cavities, less blood vessels are disrupted and thus less hemoptysis, especially middle and heavy hemoptysis cases. This is concordant with the findings in this study. Therefore, cavitary TB is an important and very reasonable risk factor for PTH.

Different TB lesions contain different amount of $M$. tuberculosis bacilli. There are about $10^{2}-10^{5}$ bacilli in a solid TB lesion, while the bacilli in a TB cavity is between $10^{7}$ and $10^{9}$ [26]. As caseous TB lesions liquefy, $M$. tuberculosis first multiply in macrophages and extracellular bacilli in the solid caseous TB lesions are suppressed. M. tuberculosis can also be neutralized easily by macrophages in the living tissue [27]. Once the caseous TB lesions liquefy, the necrotic lesions can then be exhausted to form cavities. The extracellular $M$. tuberculosis in the solid caseous TB lesions and those in the cavities then proliferate rapidly because of oxygen-rich environments. Large amounts of M. tuberculosis bacilli in the cavities connected with bronchi expelled outside by cough. In this study, we found that $79.6 \%$ of PTH cases with cavities were M. tuberculosis-positive pulmonary TB patients, while the same scenario was only $54.3 \%$ in PTH patients without cavities. The difference was statistically significant $(\mathrm{P}<0.001)$ which indicated that PTH patients with cavities were more likely $M$. tuberculosis-positive. To the best of our knowledge, this is the first study which has performed a systemic anal- 
ysis of all the five factors of PTH. The obvious limitations of this study were that the patients were mainly from southern China, especially Guangdong province and its periphery, and they were only enrolled as inpatients and with clear demographic records. Multicentric studies including more pulmonary TB patients from different sites, both inpatients and outpatients, and not only Han race patients should be considered in future studies.

In summary, PTH were more common in young (age 20 to 40 years old), male, sputum-positive, retreated and cavitary TB patients in southern China. TB patients who are prone to hemoptysis should be sensitized on the implications of TB infection and the easiness of contracting PTH. Cavitary TB patients are not only prone to hemoptysis but also M. tuberculosis-positive and thus easier to transmit M. tuberculosis. Therefore, the management of such patients needs to be strengthened to prevent the rapid and extensive transmission of TB.

\section{Conclusion}

Hemoptysis is one of the most common symptoms in patients with respiratory disease and may be a life-threatening condition. We intended to assess risk factors associated with hemoptysis in patients with tuberculosis (TB) or post-TB sequelae in a country where TB burden is relatively high, because active TB has been shown to be a major etiology of hemoptysis. Besides being a feature of active TB, hemoptysis can also be a manifestation of complications such as cavitation, fibrosis, bronchiectasis, and mycetoma. Pulmonary tuberculosis hemoptysis is related to sex, age, pathological change type, blood vessel damage spot and scope. Hemoptysis can suddenly progress into massive hemoptysis depending on the patient's status; therefore energetic cautionary measures should be implemented right from the onset of bloody expectoration. To achieve this, then a deeper understanding of the inter-related factors associated with pulmonary tuberculosis hemoptysis is needed so as to strengthen cautionary measures and standardize its diagnosis and treatment.

\section{Acknowledgements}

Tianyu Zhang was supported by the Chinese Academy of Sciences One Hundred Talents Program (Category A), the Key Program of the Chinese Academy of Sciences (KJZD-EW-L02) and the National Great Research Program of China (2013ZX10003006).

\section{Authors' Contributions}

Conceived and designed the experiments: ST, DS, TTZ, TYZ; Performed the experiments: ST, DS, TTZ, YL; Analyzed the data: ST, DS, TTZ, YL, YC, MMN, CW, TYZ; Wrote the paper: ST, DS, YL, YC, MMN, CW, TYZ.

\section{References}

[1] Pianosi, P. and Al-sadoon, H. (1996) Hemoptysis in Children. Pediatrics in Review, 17, 344-348.

[2] Jean-Baptiste, E. (2000) Clinical Assessment and Management of Massive Hemoptysis. Critical Care Medicine, 28, 1642-1647.

[3] Reechaipichitkul, W. and Latong, S. (2005) Etiology and Treatment Outcomes of Massive Hemoptysis. The Southeast Asian Journal of Tropical Medicine and Public Health, 36, 474-480.

[4] Van-Kralingen, K., Van Kralingen-Heijboer, A., Zimmerman, M. and Postmus, P. (1995) Management of Hemoptysis in a Third World City Hospital: A Retrospective Study. Tubercle and Lung Disease, 76, 344-348.

[5] Porzezińska, M., Gorzewska, A., Drozdowski, J., Sulzycka, M. and Słomiński, J. (2005) Assessment of Hemoptysis Etiology among Patients Hospitalized in Pneumonology Departament of Medical University of Gdansk in the Years 1998-2002. Polskie Archiwum Medycyny Wewnetrznej, 114, 658-663.

[6] Tsoumakidou, M., Chrysofakis, G., Tsiligianni, I., Maltezakis, G., Siafakas, N.M. and Tzanakis, N. (2006) A Prospective Analysis of 184 Hemoptysis Cases-Diagnostic Impact of Chest X-Ray, Computed Tomography, Bronchoscopy. Respiration, 73, 808-814.

[7] Soares, P.F., Teixeira, N., Coelho, F. and Damas, C. (2011) Hemoptysis-Etiology, Evaluation and Treatment in a University Hospital. Revista Portuguesa de Pneumologia (English Edition), 17, 7-14.

[8] Uzun, O., Atasoy, Y., Findik, S., Atici, A.G. and Erkan, L. (201) A Prospective Evaluation of Hemoptysis Cases in a Tertiary Referral Hospital. The Clinical Respiratory Journal, 4, 131-138.

[9] National Technical Steering Group of the Epidemiological Sampling Survey for Tuberculosis Office of the Nationwide 
Epidemiological Sampling Survey for Tuberculosis (2002) Report on Nationwide Random Survey for the Epidemiology of Tuberculosis in 2000. The Journal of the Chinese Antituberculosis Association, 24, 65-66. http://dx.doi.org/10.3969/j.issn.1000-6621.2002.02.001

[10] Hirshberg, B., Biran, I., Glazer, M. and Kramer, M.R. (1997) Hemoptysis: Etiology, Evaluation, and Outcome in a Tertiary Referral Hospital. Chest Journal, 112, 440-444.

[11] Tuberculosis Csf. (2001) Tuberculosis Diagnosis and Treatment Guidelines. Chinese Journal of Tuberculosis and Respiratory Diseases, 24, 70-74.

[12] Association, C.M. (2005) Clinical Practice Guidelines: Tuberculosis Volume. People's Medical Publishing House, Beijing, $110 \mathrm{p}$.

[13] Uplekar, M., Rangan, S., Weiss, M., Ogden, J., Borgdorff, M. and Hudelson, P. (2001) Attention to Gender Issues in Tuberculosis Control. International Journal of Tuberculosis and Lung Disease, 5, 220-224.

[14] Kolappan, C. and Gopi, P. (2002) Tobacco Smoking and Pulmonary Tuberculosis. Thorax, 57, 964-966. http://dx.doi.org/10.1136/thorax.57.11.964

[15] Bates, M.N., Khalakdina, A., Pai, M., Chang, L., Lessa, F. and Smith, K.R. (2007) Risk of Tuberculosis from Exposure to Tobacco Smoke: A Systematic Review and Meta-Analysis. Archives of Internal Medicine, 167, 335-342. http://dx.doi.org/10.1001/archinte.167.4.335

[16] Li, Y. and Gu, Z. (2010) Clinical Analysis of 90 Cases of Pulmonary Tuberculosis Hemoptysis. China Medical Herald, 5,105 .

[17] Achkar, J. and Joseph, G. (2012) Independent Association of Younger Age with Hemoptysis in Adults with Pulmonary Tuberculosis. International Journal of Tuberculosis and Lung Disease, 16, 897-902. http://dx.doi.org/10.5588/ijtld.11.0758

[18] Pérez-Guzmán, C., Vargas, M.H., Torres-Cruz, A. and Villarreal-Velarde, H. (1999) Does Aging Modify Pulmonary Tuberculosis? A Meta-Analytical Review. Chest Journal, 116, 961-967. http://dx.doi.org/10.1378/chest.116.4.961

[19] Dorshkind, K. and Swain, S. (2009) Age-Associated Declines in Immune System Development and Function: Causes, Consequences, and Reversal. Current Opinion in Immunology, 21, 404-407. http://dx.doi.org/10.1016/j.coi.2009.07.001

[20] Yuan, Y.G.W. (2012) Diagnosis and Treatment of 167 Cases of Patients with Hemoptysis Analysis. Clinical Pulmonary Medicine, 11, 2055-2055.

[21] Irodi, A. and Keshava, S. (2009) Rasmussen's Aneurysm-Undue Importance to an Uncommon Entity? British Journal of Radiology, 82, 698. http://dx.doi.org/10.1259/bjr/87990037

[22] Zellwegera, C.C., Maillarda, J.O., Christend, G. and Auberta, J.D. (2004) Successful Embolization of Rasmussen's Aneurysm for Severe Haemoptysis. Swiss Medical Weekly, 134, 47-48.

[23] Wu, X.M., Lai, Q., Chen, Y.F., Ren, Y.M., Liang, R.G. and Soong, Y.Q. (2008) Relationship between Broncho-Pulmonary Shunt and Massive Hemoptysis (a Report of 62 Cases). China Journal of Modern Medicine, 18, 939-943.

[24] Ozgül, M., Turna, A., Yildiz, P., Ertan, E., Kahraman, S. and Yilmaz, V. (2006) Risk Factors and Recurrence Patterns in 203 Patients with Hemoptysis. Türk Tüberküloz ve Toraks Derneği, 54, 243-248.

[25] Goble, M., Iseman, M.D., Madsen, L.A., Waite, D., Ackerson, L. and Horsburgh Jr., C.R. (1993) Treatment of 171 Patients with Pulmonary Tuberculosis Resistant to Isoniazid and Rifampin. New England Journal of Medicine, 328, $527-$ 532. http://dx.doi.org/10.1056/NEJM199302253280802

[26] Ma, Y. and Panyu, X. (2006) Tuberculosis. People's Health Publishing House, Beijing, 71.

[27] Sopko, D.R. and Smith, T.P. (2011) Bronchial Artery Embolization for Hemoptysis. Seminars in Interventional Radiology, 28, 48-62. http://dx.doi.org/10.1055/s-0031-1273940 
Scientific Research Publishing (SCIRP) is one of the largest Open Access journal publishers. It is currently publishing more than 200 open access, online, peer-reviewed journals covering a wide range of academic disciplines. SCIRP serves the worldwide academic communities and contributes to the progress and application of science with its publication.

Other selected journals from SCIRP are listed as below. Submit your manuscript to us via either submit@scirp.org or Online Submission Portal.
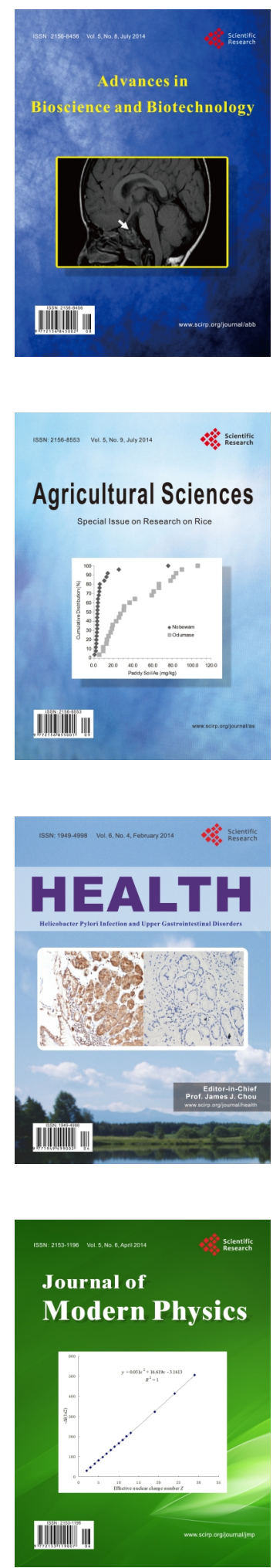
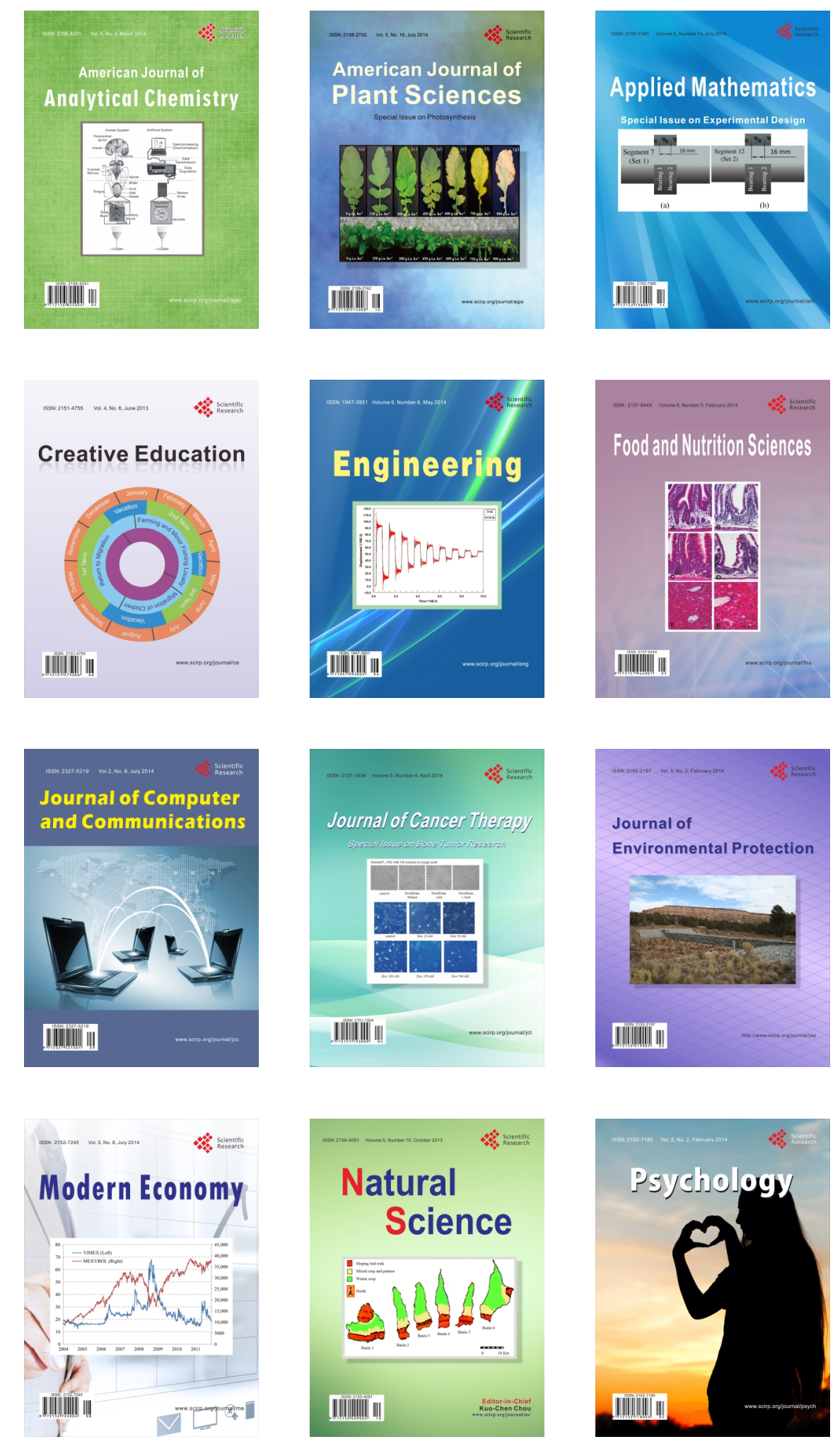